Published in final edited form as:

Curr Opin Genet Dev. 2018 February ; 48: 128-133. doi:10.1016/j.gde.2018.01.001.

\title{
Emerging roles of microRNAs in cancer
}

\author{
Ivan Vannini ${ }^{1,3}$, Francesca Fanini ${ }^{1,3}$, and Muller Fabbri, ${ }^{2, *}$ \\ ${ }^{1}$ Department of Clinical and Experimental Oncology and Hematology, Biosciences Laboratory, \\ Unit of Gene Therapy, Istituto Scientifico Romagnolo per lo Studio e la Cura dei Tumori (IRST) \\ S.r.l. IRCCS, Meldola (FC) 47014, Italy \\ 2Departments of Pediatrics and Molecular Microbiology \& Immunology, Norris Comprehensive \\ Cancer Center, Keck School of Medicine, University of Southern California, Children's Center for \\ Cancer and Blood Diseases and The Saban Research Institute, Children's Hospital Los Angeles, \\ Los Angeles, CA 90027, USA
}

\begin{abstract}
MicroRNAs (miRNAs) have emerged as important regulators of human carcinogenesis by affecting the expression of key oncogenes and tumor suppressor genes. MiRNAs elicit their function through post-transcriptional regulation of the mRNA translation into protein as well as functioning as ligands for proteic receptors called miRceptors. Our understanding of the role of miRNAs in cancer biology has enormously improved in the last few years, providing the rationale for new therapeutics. Here we discuss the most recent findings on the role of miRNAs in modulating cancer biology with a specific focus on their role of modulators of the biology of the Tumor Microenvironment both as cargo of extracellular vesicles and as extra-vesicular miRNAs.
\end{abstract}

\section{Keywords \\ miRNAs; cancer; extracellular vesicles}

\section{Introduction}

Cancer is a leading cause of death worldwide. In the last three decades, researchers have identified new cancer-specific targets that have allowed to develop anti-cancer target therapies with an important reduction of side effects. Clonal evolution of cancer cells leads

\footnotetext{
*Corresponding Author: Muller Fabbri, MD, PhD, Departments of Pediatrics and Molecular Microbiology \& Immunology, Children's Hospital Los Angeles, 4650 Sunset Blvd, Mailstop \#57, Los Angeles, CA 90027, USA. Phone: (+1) 323-361-8920; Fax: (+1) 323-361-4902; mfabbri@ chla.usc.edu.

Publisher's Disclaimer: This is a PDF file of an unedited manuscript that has been accepted for publication. As a service to our customers we are providing this early version of the manuscript. The manuscript will undergo copyediting, typesetting, and review of the resulting proof before it is published in its final citable form. Please note that during the production process errors may be discovered which could affect the content, and all legal disclaimers that apply to the journal pertain.

Authors' contributions

All the authors were involved in drafting the manuscript, revising it for intellectual content, and reading and approving the final manuscript.

Competing interests

The authors declare that they have no competing interests.
} 
to a variety of survival pathways allowing cancer cells to survive and become resistant to current treatments. Small molecules have been discovered to affect the signal transduction pathways for the survival and proliferation of cancer cells [1]. Among these, microRNAs (miRNAs) are important modifiers of transcription and translation of key regulatory proteins involved in cancer development and progression. They can inhibit or enhance the expression of oncogenes or tumor suppressor genes, therefore profoundly affecting the biology of cancer. MiRNAs belong to a large and growing family of small non-coding RNAs. They have a nucleotide sequence of 19-24 bases in length and they control gene expression by targeting specific mRNAs usually at the level of the $3^{\prime}$ untranslated region ( $3^{\prime}$-UTR) of the transcript, albeit other less conventional targeting sequences have been identified. A specific mRNA may be targeted by different miRNAs and a single miRNA can have several mRNA targets. MiRNAs are involved in the communication between cancer cells and the surrounding tumor microenvironment (TME). The interaction between tumor cells and components of TME may directly influence all aspects of cancer biology. Extracellular vesicles (EVs) are mediators of the inter-cellular cross-communication occurring within the TME $[2,3]$. EVs are characterized by a lipidic bilayer membrane analogue to the plasma membrane and a size ranging from $30 \mathrm{~nm}$ to a few microns. EVs are classified into three categories based on their size: exosomes with a size of 30-100 $\mathrm{nm}$, microvesicles with a size ranging from 100 to $1000 \mathrm{~nm}$, and large oncosomes with a size ranging 1-10 $\mu \mathrm{m}$. Furthermore, all EVs carry a complex cargo composed of proteins, mRNAs, DNA, short and long non-coding RNAs such as miRNAs that can influence the cancer growth. Exosomal miRNAs contribute to angiogenesis by modulating the expression of pro- and antiangiogenic factors. In this review, we will focus on the most important miRNAs dysregulated in tumor cells and their molecular mechanisms to regulate the expression of target genes involved in cancer growth. Finally, we will discuss the key role of miRNAs in the communication between the TME and tumor cells through EVs.

\section{miRNAs expression regulations}

miRNAs are disregulated through different mechanisms. One of these was described by Nawaz et al.. They discovered that the $m i R-4484$ is a downregulated tumor suppressor in glioblastoma and it located on chromosome 10 at fragile loci that undergoes a somatic copy number alteration through a deletion of miRNA sequence [4]. Zhou et al. showed that the amplification of the gene encoding for $\mathrm{miR}$-30d promotes cervical carcinoma growth and progression [5].

Furthermore Yin et al. demonstrated that $m i R-33 b$ is a tumor suppressor and the $\mathrm{CpG}$ island hypermethylation upstream of $m i R-33 b$ determines its downregulation in gastric cancer [6].

\section{miRNAs as oncogenes or tumor suppressor genes}

MiRNAs are often dysregulated in cancer patients compared to the normal tissue counterpart. The predicted targets of these miRNAs include both oncogenes and tumor suppressor genes. MiR-155 is upregulated in many human cancers and in more aggressive and therapy-resistant cancers as Van Roosbroeck et al. demonstrated [7]. The authors of this study observed that downregulation of $m i R-155$ achieved through a treatment with anti- 
miR-155 successfully re-sensitized tumors to chemotherapy in an in vivo orthotopic lung cancer model. Moreover, they observed a negative feedback loop between miR-155 and TP53 in 956 patients with lung cancer, acute lymphoblastic leukemia and chronic lymphocytic leukemia and showed that a signature of high levels of $m i R-155$ and low levels of TP53 is significantly correlated with shorter survival in lung cancer.

Also $m i R-9$ is frequently upregulated in several human cancers $[8,9]$. The study by $D$ 'Ippolito et al. showed that in triple-negative breast cancer, the most aggressive and undifferentiated subtype of breast cancer, $m i R-9$ has a high expression [10]. They observed that PDGFR $\beta$, a tyrosine kinase receptor for members of the platelet-derived growth factor family, induced $m i R-9$ expression, suggesting that $m i R-9$ behaves as a downstream effector of the receptor signaling. The authors discovered that $m i R-9$ targeted STARD13, a protein involved in the regulation of cytoskeletal reorganization, cell proliferation, and cell motility.

Bonci et al. dissected the role of $m i R-15, m i R-16$ and $m i R-21$ in prostate cancer patients [11]. $m i R-15$ and $m i R-16$ are often downregulated in different tumors while $m i R-21$ is upregulated [12-14]. The authors of this study observed that the concomitant loss of $m i R-15 / m i R-16$ and gain of $m i R-21$ aberrantly activate the TGF- $\beta$ signaling, increasing local invasion, distant bone marrow colonization and osteolysis by prostate cancer cells. In particular, they reported that $m i R-15 / m i R-16$ directly silences USP9X, an enzyme conducive of TGF- $\beta$ signaling activation through the deubiquitination of SMAD4, whereas miR-21 the TGF- $\beta$ signaling inhibitor SMAD7. This study establishes a new molecular circuitry for prostate cancer that involves miRNAs.

Other miRNAs with tumor suppressor function belong to the let-7 family. Spolverini et al. recently discovered that let-7b and let-7c can positively regulate the cellular expression of histone H2B (H2Bub1) in different type of tumors [15]. H2Bub1 influences chromatin function in transcription regulation and DNA repair. Cancer cells often show low levels of $\mathrm{H} 2 \mathrm{Bub1}$, and this promotes cancer progression. This positive regulation by let- $7 b$ and let-7c is achieved through the targeting of multiple mRNAs of the H2B deubiquitination machinery. In particular, let-7b and let-7c bind directly to and inhibit the mRNAs encoding for USP42 and USP44, two components of the multiple deubiquitynating enzymes (DUBs). Furthermore, these miRNAs can silence the mRNA encoding for the adapter protein ATXN7L3, which is part of the DUBs complex.

Altogether, these data suggest that miRNAs can work both as oncogenes and as tumor suppressor genes both through a direct interaction with the regulated genes and through an indirect modulation of the epigenetic regulatory systems within the cell.

Oncogenic and tumor suppressor miRNAs discussed in this paragraph are summarized in Table 1.

\section{Vesicular miRNAs in Cancer}

Recently, EVs have emerged as a prominent way of communication between cells of TME, and they are currently also accepted as mediators of carcinogenesis [16]. Interestingly, EVs from cancer cells show a specific molecular profile both in terms of content and surface 
markers, allowing their differentiation from EVs released by normal cells [17, 18]. Since Valadi et al. first demonstrated that miRNAs and other genetic material could be transferred from one cell to another by EVs, miRNAs have emerged as critical players in mediating all the processes that take place in the TME [19]. To date, very little is known about the main mechanisms of action through which vesicular miRNA elicit their functions in the recipient cells. Currently demonstrated mechanisms include the "classic" gene regulation operating through the miRNA binding to the $3^{\prime}$-UTR region of target mRNAs, and their "alternative" function mediated by the activation of miRceptors, defined as receptors for miRNAs. Yang et al. gave the first evidence of oncogenic miRNA transferring from macrophages to breast cancer cells via exosomes providing the rationale for the targeting of $m i R-223$ in M2 macrophages, or exosomal miR-223 from M2 macrophages as therapeutics. By using a coculture system they demonstrated that $m i R-223$ is shuttled from macrophages to breast cancer cells where it targets Mef2c and increases their invasiveness [20]. In the 4T1 murine breast cancer cell line, epigallocatechin gallate (EGCG), a molecule known for its anti-tumor effects, is able to suppress tumor growth. EGCG exerts its activity through the regulation of tumor-associated macrophages (TAM) in the TME, by up-regulating miR-16 in tumor cells that is subsequently transferred to TAM via exosomes. Ex vivo incubation of TAMs with EGCG-treated 4T1 exosomes led to the increase of IL-6 and TGF- $\beta$, decrease of TNF- $\alpha$, I $\mathrm{B}$ kinase $\alpha$ (IKKa) suppression and concomitant I $\mathrm{kB}$ increase [21]. Oncogenic miR-135b was significantly upregulated in exosomes from a hypoxia-resistant multiple myeloma (HRMM) cell model. Exosomal miR-135b from HR-MM cells downregulated its target factorinhibiting hypoxiainducible factor 1 (FIH-1) in endothelial cells, by targeting its $3^{\prime}$-UTR, enhancing endothelial tube formation under hypoxic condition, providing a possible target for controlling MM angiogenesis [22]. Recently, Patel et al. observed how conditioned media $(\mathrm{CM})$ from pancreatic cancer cells treated with gemcitabine (Gem-CM) gave significant chemoprotection to later gemcitabine toxicity and provided clear evidence that the majority of the chemoresistance derived from the EV fraction of Gem-CM cells. Gene expression analyses demonstrated downregulation of Deoxycytidine kinase (DCK), a gemcitabine-metabolising gene, which is directly targeted by exosome-delivered $m i R-155$, as confirmed by dual-luciferase $3^{\prime}$-UTR reporter assay [23]. Intriguingly, a recent study provided the first evidence about a cell-autonomous ability to process precursor miRNAs (pre-miRNAs) into mature form, describing how exosomes from breast cancer may transport pre-miRNAs complexed with Dicer, TRBP and AGO2 proteins [24]. Endovesicular miRNAs have been proven to function also by interacting directly with miRceptors. Fabbri et al. first identified a new mechanism of cell-to-cell communication demonstrating that exosomal $m i R-21$ and $m i R-29 a$ from non-small cell lung cancer (NSCLC) cells, were recruited in the TME by TAMs and bind to their Toll-like receptor 8 (TLR8), activating the downstream NF$\kappa B$ pathway, and increasing the secretion of inteleukin-6 (IL-6) and Tumor necrosis factor a (TNF-a) which, in turn, promote an inflammatory TME conducive to increased cancer growth and dissemination [25]. In neuroblastoma, cisplatin resistance was increased by a miR-155 mediated downregulation of Telomeric Repeat Binding Factor 1 (TERF1), a telomerase inhibitor. The underlying mechanism is a loop in which endovesicular miR-21 from neuroblastoma cells, after its binding to TLR8 in surrounding TAMs, up-regulates $m i R-155$ which is then secreted in TAM-derived EVs, and transferred back to neuroblastoma cells where it silences TERF1 and increases resistance to chemotherapy [26]. 
The vesicular miRNAs mechanisms described in this paragraph are shown in Figure 1, whereas the vesicular miRNAs discussed in this article are summarized in Table 2.

\section{Extra-Vesicular miRNAs in Cancer}

It was previously demonstrated that miRNAs circulate in plasma and serum in a highly stable cell-free form, with a profile that can be altered in cancer and other diseases [27-29]. The high concentration of RNase activity in the bloodstream has prompted the scientific community to try to explain the mechanism underlying the surprising stability of circulating miRNAs (c-miRNAs). Initially, the release of c-miRNAs in membrane-bound vesicles (exosomes and microvesicles) with protective function was considered the most common form in which miRNAs were found in circulation. Surprisingly, this assumption was challenged by Wang et al. who found that biggest fraction of extracellular miRNAs was actually located outside of EVs [30]. Several studies have further supported this discovery. In 2011, Arroyo et al. showed that endovesicular miRNAs represent only a minor fraction of c-miRNAs, and that unexpectedly the majority of them co-fractionated with protein complexes, rather than with vesicles, protecting c-miRNAs from plasma RNases. These results significantly revised our knowledge about the physiological state and nature of cmiRNAs stability, with consequent backlashes on future studies about miRNAs as biomarkers and the function of extracellular miRNAs [31]. To date, less is known about the role and mechanism of action of Ago2/c-miRNA complexes, and it is also quite unclear whether they are released from viable cells and can be directed to recipient cells. C-miRNAs have also been found in association with lipoprotein such as low-density lipoprotein (LDL), or high-density lipoprotein (HDL) [32], which are able to carry nucleic acids and are often used as agents for gene delivery [33] (Figure 2). In patients with familial hypercholesterolemia, Vickers et al. identified for the first time a specific signature of miRNAs (including miR-223, miR-105 and miR-106a) in HDL-miRNA complexes. Interestingly, the delivery of these HDL-miRNAs allowed a functional downregulation of their corresponding mRNA targets in cultured hepatocytes, suggesting the functional importance of these lipoprotein/c-miRNAs complexes [34]. In summary, while the full spectrum of mechanisms of action of c-miRNAs is still far from being elucidated, there is little doubt that they are involved in shaping the biology of cancer and of the TME.

\section{Summary and Future Directions}

MiRNAs regulate human carcinogenesis and affect the biology of the TME. They are also key mediators of inter-cellular communication both as c-miRNAs and as cargo of EVs. While the plethora of targets of frequently dys-regulated miRNAs has been the object of several studies, one of the biggest challenges is a better understanding of the selective mechanisms of secretion and delivery of extracellular miRNAs to the cells of the TME. Also, the specificity of uptake (if it exists) will improve out efforts of using miRNAs as therapeutics alone or, more likely, in combination with existing anti-cancer therapies. Finally, a broader study of the pattern of miRceptors and their topographic distribution within the cells of the TME will expand our understanding of this new fascinating branch of cancer biology and will extend our anti-cancer weaponry with the goal of increasing the number of saved lives of patients diagnosed with cancer. 


\section{Acknowledgments}

Dr. Fabbri is a St. Baldrick Foundation's Scholar and is supported by the NIH/NCI grant R01CA215753, a Hyundai Hope on Wheels grant, a Jean Perkins Foundation grant, the Nautica Malibu Triathlon Funds, a STOP Cancer grant, the Hugh and Audy Lou Colvin Foundation grant, an Alex's Lemonade Stand Foundation grant, and the award number P30CA014089 from the National Cancer Institute.

\section{References}

1. Hoelder S, Clarke PA, Workman P. Discovery of small molecule cancer drugs: Successes, challenges and opportunities. Mol Oncol. 2012; 6:155-176. [PubMed: 22440008]

2. Raposo G, Stoorvogel W. Extracellular vesicles: exosomes, microvesicles, and friends. J Cell Biol. 2013; 200:373-83. [PubMed: 23420871]

3. Di Vizio D, Morello M, et al. Large oncosomes in human prostate cancer tissues and in the circulation of mice with metastatic disease. Am J Pathol. 2012; 181:1573-84. [PubMed: 23022210]

4. Nawaz Z, Patil V, et al. Impact of somatic copy number alterations on the glioblastoma miRNome: miR-4484 is a genomically deleted tumour suppressor. Mol Oncol. 2017; 11(8):927-944. [PubMed: 28378523]

5. Zhou Y, Hao Y, et al. Amplification and up-regulation of MIR30D was associated with disease progression of cervical squamous cell carcinomas. BMC Cancer. 2017; 17(1):230. [PubMed: 28356144]

6. Yin H, Song P, et al. DNA Methylation mediated down-regulating of MicroRNA-33b and its role in gastric cancer. Sci Rep. 2016; 6:18824. [PubMed: 26729612]

7•. Van Roosbroeck K, Fanini F, et al. Combining Anti-Mir-155 with Chemotherapy for the Treatment of Lung Cancers. Clin Cancer Res. 2017; 23:2891-2904. The first report on the role of TP53 as a direct target of miR-155 and the first demonstration that an anti-miR-155 treatment is effective in NSCLC in an orthotopic murine model. [PubMed: 27903673]

8. Ma L, Young J, et al. miR-9, a MYC/MYCN-activated microRNA, regulates E-cadherin and cancer metastasis. Nat Cell Biol. 2010; 12:247-56. [PubMed: 20173740]

9. Gwak JM, Kim HJ, et al. MicroRNA-9 is associated with epithelial-mesenchymal transition, breast cancer stem cellphenotype, and tumor progression in breast cancer. Breast Cancer Res Treat. 2014; 147:39-49. [PubMed: 25086633]

10•. D'Ippolito E, Plantamura I, et al. miR-9 and miR-200 Regulate PDGFR $\beta$-Mediated Endothelial Differentiation of Tumor Cells in Triple-Negative Breast Cancer. Cancer Res. 2016; 76:5562-72. A seminal paper showing the role of miR-9 and miR-200 in the regulation of endothelial differentiation in triple negative breast cancer. [PubMed: 27402080]

11••. Bonci D, Coppola V, et al. A microRNA code for prostate cancer metastasis. Oncogene. 2016; 35:1180-92. An outstanding study that identifies a specific signature of miRNAs involved in the different aspects of the biology of prostate cancer metastasis. [PubMed: 26073083]

12. Cimmino A, Calin GA, et al. miR-15 and miR-16 induce apoptosis by targeting BCL2. Proc Natl Acad Sci U S A. 2005; 102:13944-9. [PubMed: 16166262]

13. Lovat F, Fassan M, et al. miR-15b/16-2 deletion promotes B-cell malignancies. Proc Natl Acad Sci U S A. 2015; 112:11636-41. [PubMed: 26324892]

14. Janaki Ramaiah M, Lavanya A, et al. MiR-15/16 complex targets p70S6 kinase 1 and controls cell proliferation in MDA-MB-231 breast cancer cells. Gene. 2014; 552:255-64. [PubMed: 25261849]

15. Spolverini A, Fuchs G, et al. let-7b and let-7c microRNAs promote histone H2B ubiquitylation and inhibit cell migration by targeting multiple components of the $\mathrm{H} 2 \mathrm{~B}$ deubiquitylation machinery. Oncogene. 2017; 187

16. Peinado H, Aleckovic M, Lavotshkin S, et al. Melanoma exosomes educate bone marrow progenitor cells toward a pro-metastatic phenotype through MET. Nat Med. 2012; 18:883-891. [PubMed: 22635005]

17. Cocucci E, Meldolesi J, et al. Ectosomes and exosomes: shedding the confusion between extracellular vesicles. Trends Cell Biol. 2015; 25:364-372. A comprehensive overview on the classification of extracellular vesicles and its limits. [PubMed: 25683921] 
18. Wieckowski E, Whiteside TL, et al. Human tumor-derived vs dendritic cell-derived exosomes have distinct biologic roles and molecular profiles. Immunol Res. 2006; 36:247-254. [PubMed: 17337785]

19. Valadi H, Ekstrom K, Bossios A, et al. Exosome-mediated transfer of mRNAs and microRNAs is a novel mechanism of genetic exchange between cells. Nat Cell Biol. 2007; 9:654-659. [PubMed: 17486113]

20. Yang M, Chen J, et al. Microvesicles secreted by macrophages shuttle invasion-potentiating microRNAs into breast cancer cells. Molecular Cancer. 2011; 10:117. [PubMed: 21939504]

21. Jang JY, Lee JK, et al. Exosome derived from epigallocatechin gallate treated breast cancer cells suppresses tumor growth by inhibiting tumor-associated macrophage infiltration and M2 polarization. BMC Cancer. 2013; 13:421. [PubMed: 24044575]

22. Umezu T, Tadokoro H, et al. Exosomal miR-135b shed from hypoxic multiple myeloma cells enhances angiogenesis by targeting factor-inhibiting HIF-1. Blood. 2014; 11:3748-57.

23•. Patel GK, Khan MA, et al. Exosomes confer chemoresistance to pancreatic cancer cells by promoting ROS detoxification and miR-155-mediated suppression of key gemcitabinemetabolising enzyme, DCK. Br J Cancer. Feb.2017 28:609-619. An important study on the role of miR-155 in the chemo-resistance of pancreatic cancer.

24. Melo SA, Sugimoto H, et al. Cancer exosomes perform cell-independent microRNA biogenesis and promote tumorigenesis. Cancer Cell. 2014; 26:707-721. [PubMed: 25446899]

25. Fabbri M, Paone A, et al. MicroRNAs bind to Toll-like receptors to induce prometastatic inflammatory response. Proc Natl Acad Sci U S A. 2012; 109:E2110-6. [PubMed: 22753494]

26••. Challagundla KB, Wise PM, et al. Exosome-mediated transfer of microRNAs within the tumor microenvironment and neuroblastoma resistance to chemotherapy. J Natl Cancer Inst. 2015; 107(7) pii: djv135. The first report that miR-miRceptor interactions between cancer cells and TAMs affect cancer resistance to chemotherapy through the shuttling of exosomal miRNAs.

27. Mitchell PS, Parkin RK, et al. Circulating microRNAs as stable blood-based markers for cancer detection. Proc Natl Acad Sci USA. 2008; 105:10513-10518. [PubMed: 18663219]

28. Chen X, Ba Y, et al. Characterization of microRNAs in serum: A novel class of biomarkers for diagnosis of cancer and other diseases. Cell Res. 2008; 18:997-1006. [PubMed: 18766170]

29. Gilad S, Meiri E, et al. Serum microRNAs are promising novel biomarkers. PLoS ONE. 2008; 3:e3148. [PubMed: 18773077]

30. Wang K, Zhang S, et al. Export of microRNAs and microRNA-protective protein by mammalian cells. Nucleic Acids Res. Nov.2010 38:7248-59. [PubMed: 20615901]

31. Arroyo JD, Chevillet JR, et al. Argonaute 2 complexes carry a population of circulating microRNAs independent of vesicles in human plasma. Proc Natl Acad Sci U S A. Mar.2011 108:5003-8. [PubMed: 21383194]

32. Babin PJ, Gibbons GF. The evolution of plasma cholesterol: direct utility or a "spandrel" of hepatic lipid metabolism? Prog Lipid Res. 2009; 48:73-91. [PubMed: 19049814]

33. Kim SI, Shin D, et al. Systemic and specific delivery of small interfering RNAs to the liver mediated by apolipoprotein A-I. Mol Ther. 2007; 15:1145-1152. [PubMed: 17440441]

34. Vickers KC, Palmisano BT, et al. MicroRNAs are transported in plasma and delivered to recipient cells by high-density lipoproteins. Nat Cell Biol. 2011; 13:423-433. [PubMed: 21423178] 


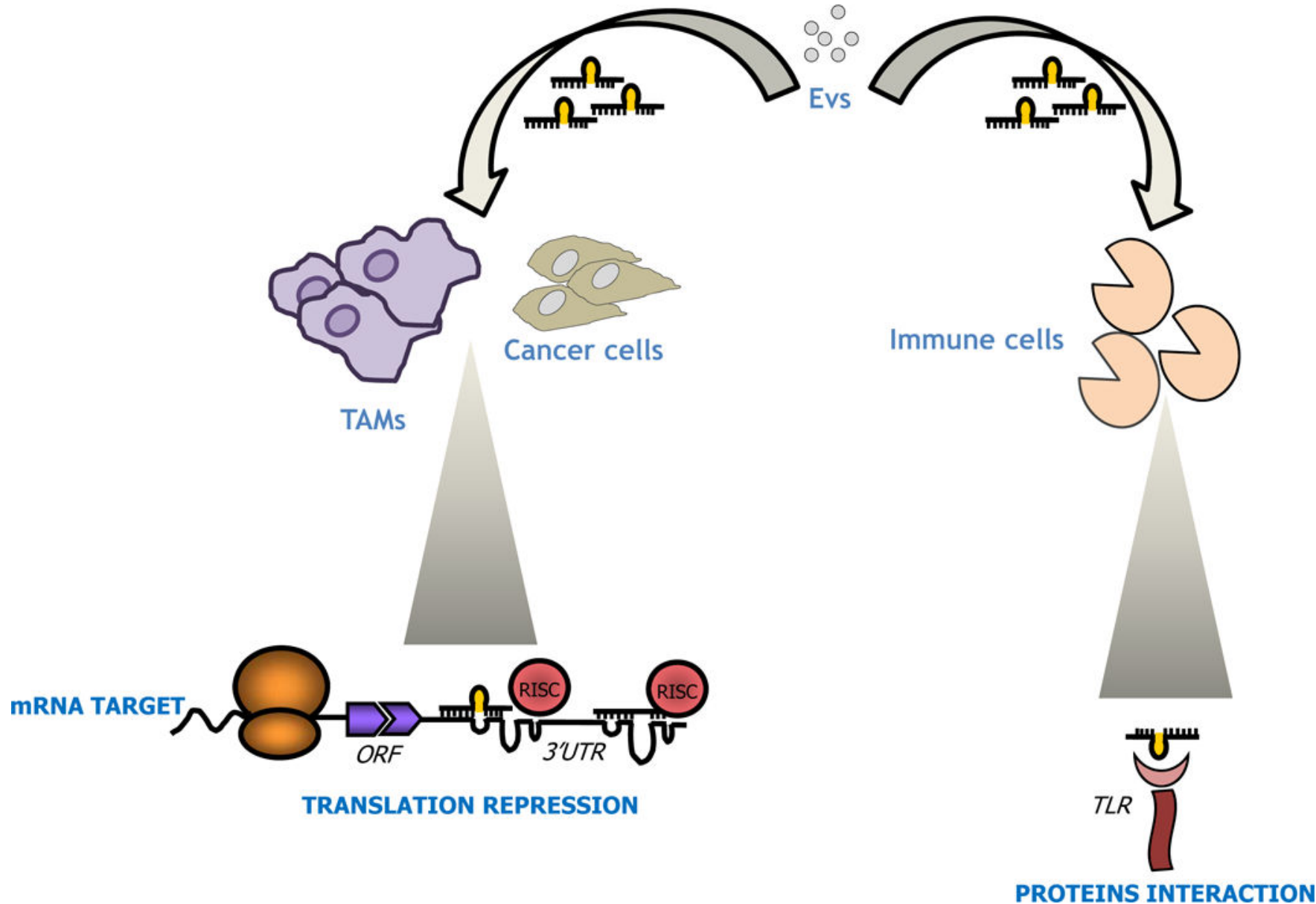

Figure 1. Mechanisms of action of Vesicular miRNAs

MiRNAs released by extra-cellular vesicles can interact with cells of the Tumor

Microenvironment by binding to the $3^{\prime}$-UTR region of target mRNAs, or by binding to- and activating Toll-like Receptor 8 (TLR8) in surrounding cells of the immune system. 


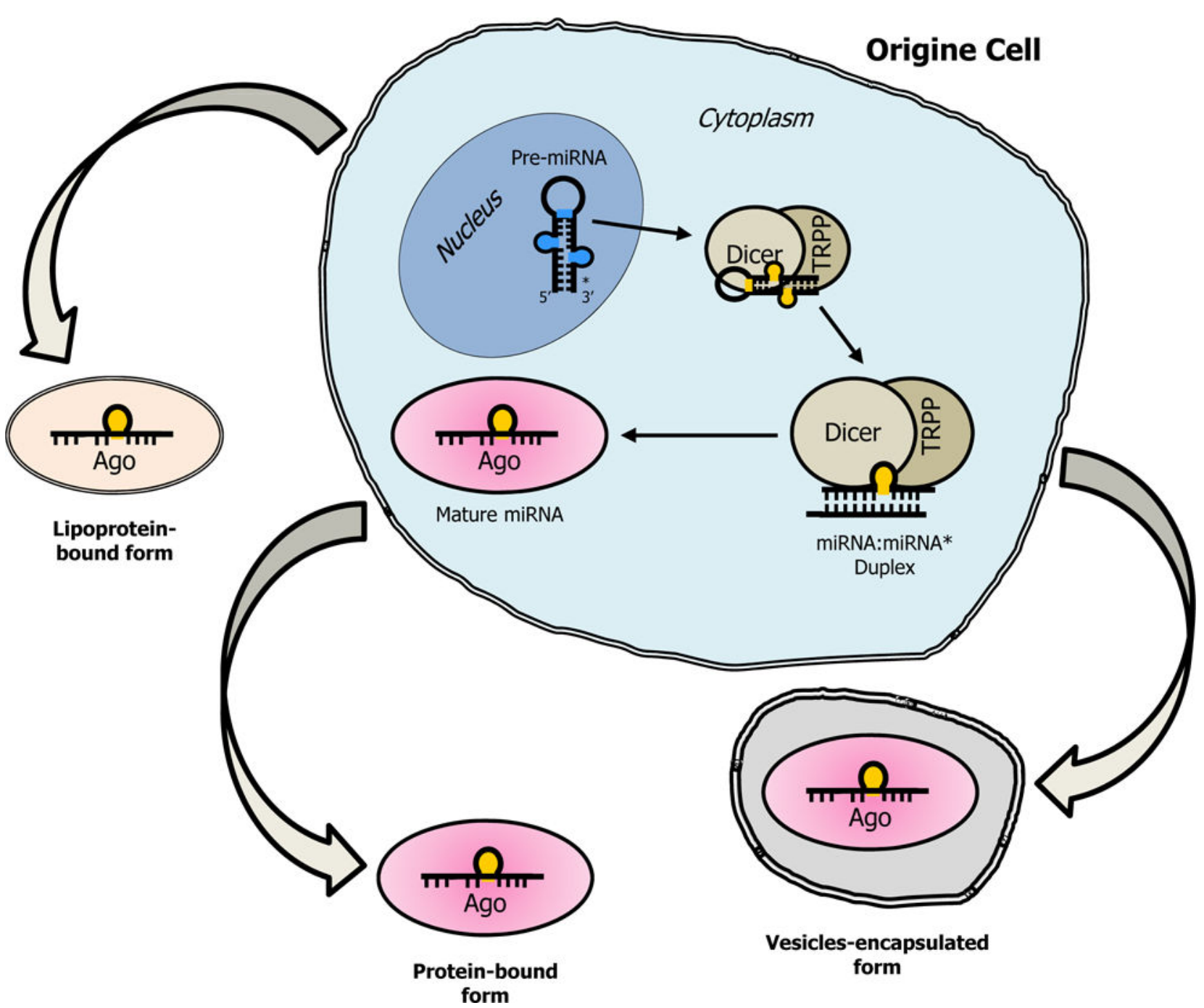

Figure 2. The fate of circulating miRNAs

MiRNAs can be released by cells in the surrounding microenvironment into three distinct forms: included in membrane-bound vesicles, associated with proteic complexes or in association with lipoproteins. 
Table 1

Principal known

miRNAs with Oncogene or Tumor suppressor role

\begin{tabular}{|c|c|c|c|c|}
\hline miRNA & Tumor function & Cancer type & Target molecule & Author \\
\hline miR-155 & Oncogene & Lung cancer, Leukemia & TP53 & {$[7]$} \\
\hline miR-9 & Oncogene & Breast cancer & STARD13 & {$[10]$} \\
\hline miR-21 & Oncogene & Prostate cancer & SMAD7 & {$[11]$} \\
\hline miR-15 & Tumor suppressor & Prostate cancer & USP9X & {$[11]$} \\
\hline miR-16 & Tumor suppressor & Prostate cancer & USP9X & {$[11]$} \\
\hline let-7 & Tumor suppressor & Lung cancer, Breast cancer & USP42, USP44, ATXN7L3 & {$[15]$} \\
\hline
\end{tabular}


Table 2

Vesicular miRNAs involved in cancer

\begin{tabular}{|c|c|c|c|c|}
\hline miRNA & Target Molecule & Involved Pathway & Involved Mechanism & Author \\
\hline miR-223 & Mef2c & Mef2c-b-catenin & $3^{\prime}$ UTR targeting & {$[20]$} \\
\hline miR-16 & IKKa & NF- $\mathrm{kB}$ & $3^{\prime}$ UTR targeting & {$[21]$} \\
\hline miR-135b & FIH-1 & angiogenesis & $3^{\prime}$ UTR targeting & {$[22]$} \\
\hline miR-155 & DCK & acquired chemoresistance & $3^{\prime}$ UTR targeting & {$[23]$} \\
\hline miR-21 & NF- $\mathrm{kB}$ & inflammation & TLR8 interaction & {$[25]$} \\
\hline miR-29 & NF- $\times B$ & inflammation & TLR8 interaction & {$[25]$} \\
\hline miR-21/miR-155 & TERF1 & Cisplatin resistance & TLR8 interaction & {$[26]$} \\
\hline
\end{tabular}

